\title{
Developing a Mobile Application via Bluetooth Wireless Technology for Enhancing Student-Instructor Communication
}

\author{
doi:10.3991/ijim.v3i3.830 \\ Dr. Sahar Idwan \\ The Hashemite University, Zarqa, Jordan
}

\begin{abstract}
A Bluetooth mobile application is developed to enhance student-instructor interaction during delivery of university courses. The paper presents Mobile application via Bluetooth wireless technology (MAvBT) consists of a server installed on a Bluetooth enabled computer, mobile application installed on student's mobile phones, and a website that enables the instructors to edit materials and the demonstrator to obtain appropriate feedback and relevant reports. Students' mobile phones are connected to the server which utilize the MAvBT application implemented to facilitate communication with instructors outside office hours as well as information retrieval with minimum time and from anywhere ranging from $100 \mathrm{~m}$ up to $1 \mathrm{~km}$. Experimental results show that the proposed system offers fast and low-cost interaction with minimum administrative offers.
\end{abstract}

Index Terms-Bluetooth, MAvBT, mobile application, wireless technology

\section{INTRODUCTION}

Bluetooth is a wireless communication protocol used to communicate two or more other Bluetooth-capable devices. It is similar to any other communication protocol such as HTTP, FTP, SMTP, or IMAP in a way that it has a client-server architecture where the one who initiates the connection is the master and the other who receives the connection is the slave. There are two communication protocols available in the mobile device or PDA depending on the cost of establishing the connection. The first one is a non free connection protocol that needs to pay for every loaded and uploaded byte between the client and the server. It is similar to the HTTP protocol that uses GPRS profile. The other one is a free connection protocol that establishes the connection between server and client without any need to be connected to a large provider like the mobile communication companies. The second protocol is used with our system. MAvBT allows the students and the instructors to communicate with each other in different ways without limitation such as capacity of labs, costs, type or numbers of recipients within the domain. The instructor is able to publish notes, exam, and homework's. The students establish the connection by using the Bluetooth and receiving the required information based on his/her request. The student is able to retrieve the evaluation form of the instructor fill it and send it back by using the MAvBT.
This paper is organized as follows. In Section II, we briefly review the related work via Bluetooth connectivity. The developed system is introduced in Section III. The implementation of the system by using the Java standard edition (J2SE) for the server application and Java micro (J2ME) edition for the mobile application is presented in Section IV. Section V provides the conclusion of the paper.

\section{RELATED WORK}

Reference [1] presented Bluetooth as an industrial specification for wireless Personal Area Networks that provides a way to connect and exchange information between devices, such as mobile phones, laptops, PCs, printers and digital cameras via a secure, low-cost and globally unlicensed short-range radio frequency

Building a wireless information system by using the Bluetooth wireless technology described in [2]. A Bluetooth-enabled client-sever system is under development to conduct assignment during the lectures.

In [3], they introduced a multimedia message transmitter tool that provides businesses with the opportunity to use Bluetooth wireless connectivity delivering the relevant information to relevant people in the right places and time. It empowers any business to market their services or products for unlimited time with a one-time fee.

The opportunity of using cellular phone for educational purposes specifically for teaching and learning mathematics examined in [4]. The students can update or share their learning process without any needs to be in the same place by using emails, logging, and MMS. This approach is expensive since we need to pay the cost of the sending and receiving messages.

A mobile web services solution to support education activities is proposed in [5]. Their work utilizes the mobile web technologies and SMS.

The use of classroom response to improve the classroom interactivity examined in [6].

In this paper, we introduced a communication tools for sharing data through the mobile phone by using the Bluetooth ID. Our system provides benefits to both students and professors. It reduces the pressure on the labs by allowing the students to access their information at any time, anywhere (in the range of his/her department), and from his own tool (mobile phone device). 


\section{ThE PROPOSED MAvBT SYSTEM}

In the revolution of mobile and wireless technology and using it in different environment such as universities, schools and marketing, we developed MAvBT. Fig. 1 presents the architecture of the system and the sequence of the operation. Our system consists of one application server, one client application and a website that enables the instructors to edit materials and share their own course documents and information at any time and the demonstrator to obtain appropriate feedback and relevant reports. The application server that utilizes the MAvBT is dedicated for handling client requests and responding to them. The client application is designed to be stored on the student's mobile phones, which enable them to access the required information via MAvBT. Bluetooth Radio Technology represents the transmission media between server and client.

The student uses the mobile application to send a message via the Bluetooth to the server that utilizes the MAvBT application to trigger the appropriate function. The MAvBT in the server side receives the message analyzes it, and returns the required information to the mobile application.

MAvBT is considered as an enhancement for the student portal system and solves the following problems:

1. When the labs are busy, it will be easy for the students to reach their information such as exam table, or classroom number through his/her phone device and within a range of the faculty.

2. Receiving notes with or without the instructor's absences.

3. Receiving shared files that the instructor need to distribute through their phone devices.

4. Ability to retrieve their general information such as grades system or current courses, and the general announcements.

5. The ability to make the instructor assessment through the Bluetooth by using a stand alone mobile application.

\section{A. Security}

In order to provide information confidentially, the Bluetooth system provides security measures at both the application layer and the link layer that presented in [7] and [8]. These measures are appropriate for a peer environment such that in each Bluetooth unit, the authentication and encryption routines are implemented in the same way. Four different entities are used for maintaining security at the link layer: a public address that is unique for each user, two secret keys, and a random number that is different for each new transaction. Ref. [9] discussed that the Bluetooth is a highly secure system for the following reasons:

1. Every Bluetooth device is given a 48-bit address that uniquely identifies the device. Every Bluetooth device on earth will have a unique address.

2. When one device wants to communicate with another device, the second device is authenticated.

3. Data on the channel is encrypted so that only the intended recipients can receive it.

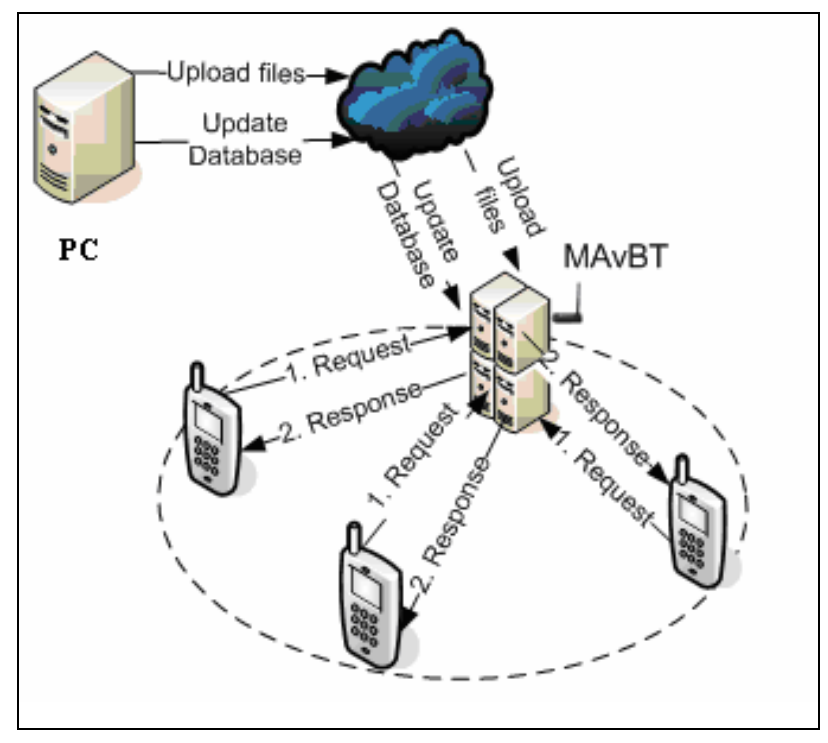

Figure 1. Architecture of the MAvBT

4. Every Bluetooth device has a random number generator; these numbers are used for authentication.

5. A frequency-hopping scheme provides built in security. Only those devices that know the hopping sequence can decode the data sent by the master.

The MAvBT used these technologies to send secure message that reduce the possibility of intruding the mobile with viruses.

\section{IMPLEMENTATION}

Our system was implemented by using the Active Server Pages (ASP.NET) in [10] with C-Sharp (C\#.NET) Technologies in [11] and [12] to implement the application servers, and Java 2 Micro Edition (J2ME) Technology in [13], [14], and [15] to implement the client application. The web administration page which is designed for the instructor's use to publish any information for his/her students' runs in the command prompt program (Win-32 application) or DOS-based application. Fig. 2 presents sample from the admin page, this page is used to publish an assignment to a specific course or course section.

We download the client application in the phone that allows the student to access the required information after inserting the user name and the password. Then the main list in Fig. 3 contains several options such as:

- Instructors: Where the student can get any related information about the instructor.

- Announcements: Where he/she can find any announcement dedicated to him/her, or to a specific course section.

- Search Mode: If he/she could not find which instructor instructs a specific course.

- Student: he/she can access the information related to $\operatorname{him} /$ her. 


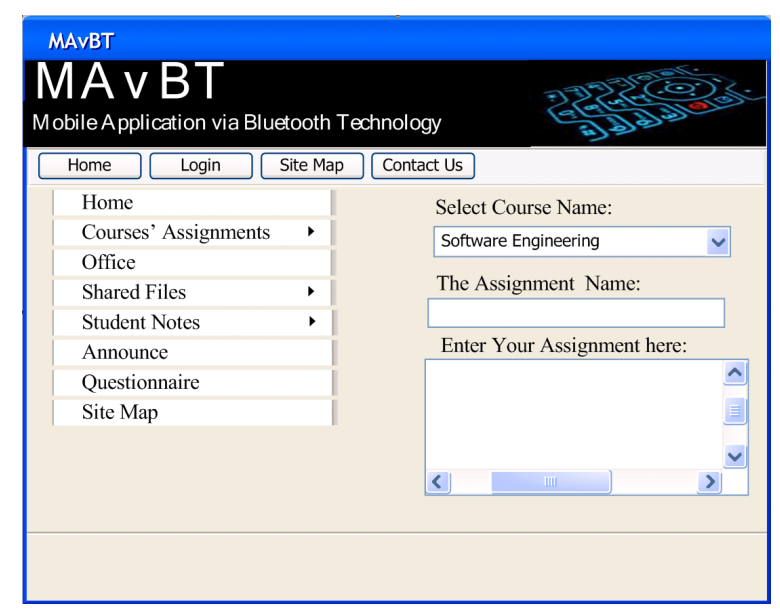

Figure 2. Admin Page

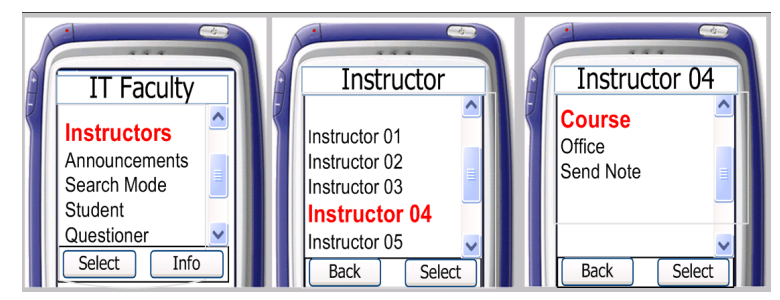

Figure 3. Client Application

As appears in Fig. 3, after selecting the instructors, a list of the instructor InstructorList appears so you can choose any instructor. After you select the instructor a new window appears contains the following selections:

- Course: To find out all courses for the selected instructor.

- Office: To find out the information related to the instructor's office (Office Hours, Office Room).

- Send Note: To send a note or complain for this instructor.

MAvBT helps to evaluate the instructor. The evaluation form can be downloaded into the client application. After answering all the questions MAvBT asks the student to press the submit button, then the application will establish a connection to the server to do the following:

- Submitting the answers to the Server's Database.

- Pointing the selected instructor to be evaluated once by the student, avoiding the reassessment of the instructor by the same student.

- Retrieve the rest of the instructor's assessment forms, in case the student wants to continue evaluating other instructors.

\section{A. Testing}

Table 1 shows the Bluetooth dongles information that is used to test the MAvBT. A "dongle" is a small hardware device that helps the computer to authenticate a piece of software or the identity of a user [16]. It connects with a computer's USB port and enables it to communicate with the other Bluetooth-enabled devices. It is available in one of three classes: Class 1 covers up to 100 meter, Class 2 covers up to 10 meter, and Class 3 covers up to 1 meter. In our experiments, we used Class 1 and Class2.
TABLE I.

BLUETOOTH DONGLE INFORMATION

\begin{tabular}{|c|c|c|c|c|}
\hline $\begin{array}{c}\text { Bluetooth } \\
\text { Dongle } \\
\text { name }\end{array}$ & $\begin{array}{c}\text { Instant } \\
\text { Connections }\end{array}$ & Class & Range & $\begin{array}{c}\text { No. of } \\
\text { sending } \\
\text { Files }\end{array}$ \\
\hline SAM sync & Up to 7 & Class 2 & 10 meter & 1 \\
\hline MSI & Up to 7 & Class 1 & 100 meter & 5 \\
\hline
\end{tabular}

Two kinds of questionnaire are distributed to the students and instructors to evaluate the MAvBT based on enhancing the education and improving the learning outcomes. The first one distributed among two hundred different students contains a variety of questions such as: age, gender, owning mobile with Bluetooth technology, how frequently using the Bluetooth technology within the university, can you use the Bluetooth technology, did the mobile support the java applications technology, the availability of the labs, and are you able to contact the instructtors in their office hours. Fig. 4 shows the results to selected questions of the first questionnaire. We found that the percentage of student's phone that supports Bluetooth technology is $89 \%$, while the rest does not. When we searched about the percentage of the students who use Bluetooth technology frequently we found, 71\% do, while $29 \%$ don't. The mobile phones that support Java applications technology which is the core of the client side is $67 \%$. When we asked about the availability of the labs we found that only $7 \%$ said they are always available, and $93 \%$ said they are rarely or sometimes available.

$64 \%$ of the students that contact the instructors (A lot, Sometimes, And Rarely) find it hard to contact their instructors in their office hours.

The second questionnaire distributed among thirty different instructors containing several of questions such as: did you use the e-mail or the paper to send the information to the students, did the students get the most recent information, and did you notice an increase in the number of students that do the evaluation for the instructors by using MAvBT.

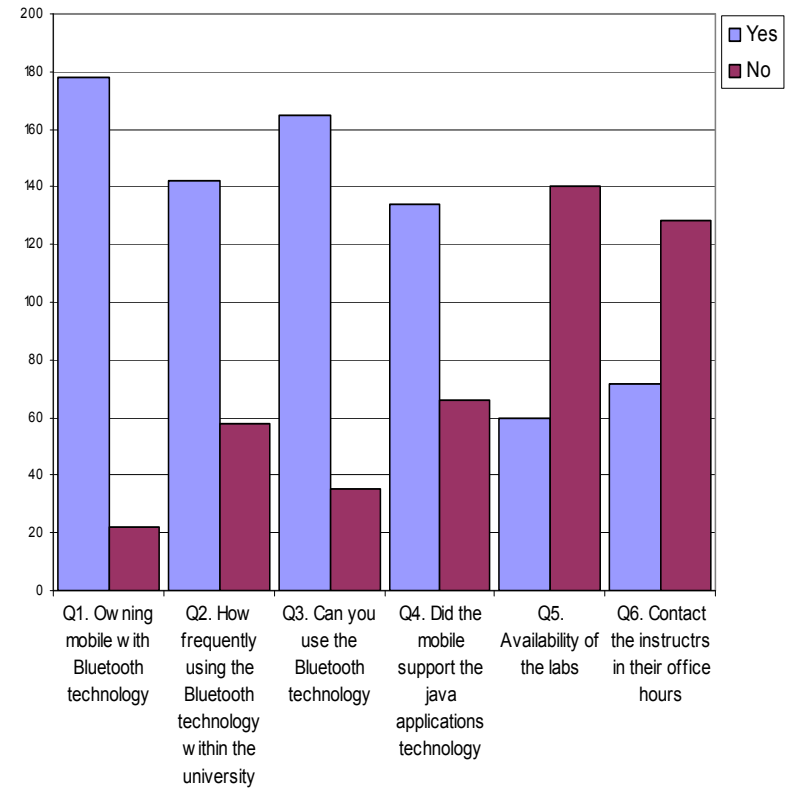

Figure 4. Evaluation of MAvBT based on Student Responses 


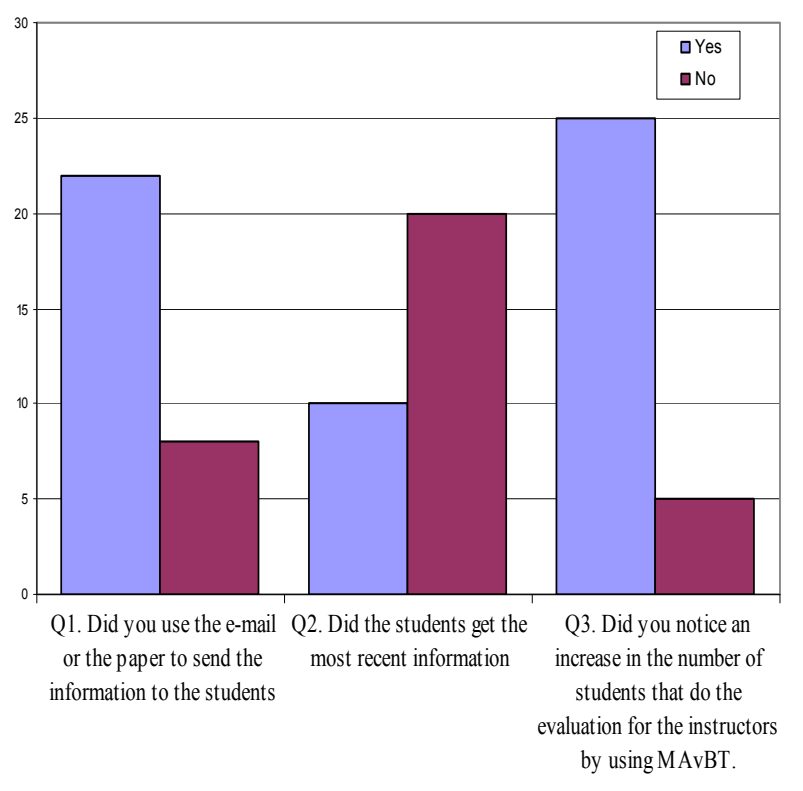

Figure 5. Evaluation of MAvBT based on Instructors Responses

Fig. 5 shows the results of the second questionnaire. Most of instructors use the e-mails and the paper to communicate with their students. They were excited about the MAvBT, because it was efficient, effortless, and cheap for the student and it increased the number of students that can be reached by the instructors.

\section{CONCLUSION}

A wireless system which may enhance studentinstructor interaction through utilization of Bluetooth technology is presented. The proposed system was based on Java standard edition (J2SE) for the server application and Java Micro (J2ME) edition for the mobile application. The MAvBT was implemented as a tool for interaction between students and educators as it offers a low-cost solution since it avoids use of complex network infrastructure. The proposed system enabled students to retrieve information at any time from anywhere. A simple user-friendly administrator web page, designed for instructor's use only, was used to assist the instructor to publish information to his/her students. Test results showed that the system provides flexible, easy and a viable solution for using mobile technology as it fulfill needs of students as well as the educators.

\section{REFERENCES}

[1] Hopkins, B. and Antony, R. (2003) Bluetooth for Java. Berkeley, CA: Apress

[2] Davidrajuh, R, "Exploring the Use of Bluetooth in Building Wireless Information Systems, " International Journal of Mobile Communications, Vol. 5, No. 1, pp. 1-10, 2007 (doi:10.1504/IJMC.2007.011486)

[3] Idwan, S., Alramouni, S., Al-Adhaileh, M. and Al-Khasawneh, A., "Enhancing Mobile Advertising via Bluetooth Technology," International Journal of Mobile Communications, v. 6, n. 5, p. 587-597, July 2008 (doi:10.1504/IJMC.2008.019323)

[4] Yerulshalmy, M., Ben-Zaken, O. (2004), "Mobile Phones in education: the case of mathematics," The Institute for Alternatives in Education, University of Haifa, available at: http://construct.haifa.ac.il/michalyr/cellular\%20report.pdf , 2004.

[5] M. Lytras, E. Sakkopoulos, and A. Tsakalidis, "Adaptive Mobile Web Services Facilitate Communication and Learning Internet Technologies," in IEEE Transactions on Education, special issue on "Mobile Technology for Education" edited by Keng Siau, Vol 42, No 2, pp. 208-215, 2006

[6] Siau, K., Sheng, H. and Nah, F., "Use of Classroom Response System To Enhance Classroom Interactivity," IEEE Transactions on Education, Vol. 49, No. 3, pp. 398-403.,2006 (doi:10.1109/TE.2006.879802)

[7] Direct Marketing Association (UK) Ltd, "Mobile Marketing Best Practice Guidelines,” $\quad \underline{\text { http://www.dma.org.uk/content/Pro- }}$ BestPractice.asp, 2005

[8] Miller, M. (2001), Discovering Bluetooth, SYBEX Inc.

[9] Dreamtech Software Team, WAP, Bluetooth, and $3 G$ Programming: Cracking the Code, Hungry Minds, Inc., 2002

[10] Francis, Rob, and Jamsa, Kris Rob Francis, Rescued by Active Server Pages and Asp.Net, Onword Pr, 2002

[11] Jason Price , and Mike Gunderloy, Mastering Visual C\#.NET, Sybex; 1st edition (August 20, 2002)

[12] Mickey Williams, Microsoft Visual C\#.NET (Core Reference), Microsoft Press; (March 27, 2002)

[13] Eric Giguere, Java 2 Micro Edition: Professional Developer's Guide, John Wiley \& Sons

[14] O'Connor, Java 2 Micro Edition Programming, Wiley Hungry Minds, 2002

[15] Michael Kroll, and Stefan Haustein, Java 2 Micro Edition (J2ME) Application Development, Pearson Education; 1st edition (June 25, 2002)

[16] SafeCom Technologies Limited, Bluetooth, User's Manual Class 1 Bluetooth USB Dongle, 2005

\section{AUTHORS}

Sahar Idwan is with the Computer Science Department in Prince Al-Hussein Bin Abdullah II of Information Technology, Hashemite University, P.O. Box 150459, Zarqa 13115, Jordan (E-mail: sahar@hu.edu.jo; sahar700@hotmail.com)

Manuscript received 4 February 2009. Published as submitted by the author. 